\title{
LAMBERTIAN INVARIANCE AND APPLICATION TO THE PROBLEM OF OPTIMAL FIXED-TIME IMPULSIVE ORBITAL TRANSFER
}

\author{
FANG-TOH SUN \\ National Tsing Hua University, Hsinchu, Taiwan, Republic of China \\ and \\ NGUYEN X. VINH $\ddagger$ \\ The University of Michigan, Ann Arbor, MI 48104, U.S.A
}

(Received 6 January 1983)

\begin{abstract}
To develop an efficient technique for the numerical solution of the Lambert problem, a new Lambertian invariant, the ratio of two invariable lengths, is proposed to replace the Gauss ratio of two areas as the main iterative variable in the time equation, and iteration schemes are devised for fast convergence under various conditions. The problem of minimum fuel 2-impulse transfer between two coplanar circular orbits under fixed time of transfer is then analyzed and numerically solved by the technique developed. The use of multi-revolution to improve the solution in the long duration case is outlined and numerically illustrated; and the two cases, wherein the two circular orbits are in the same direction of motion (uni-rotating), or in oppositedirections (counter-rotating) are distinguished and compared. Finally, by extending the study from 2 -impulse to 3 -impulse transfer a global synthesis of the various possible types of fixed time optima under different transfer conditions is briefly presented.
\end{abstract}

\section{INTRODUCTION}

The problem of minimum fuel impulsive orbital transfer in the time-free case has been extensively analyzed in the published literature, and a full account of the state of the art is found in the excellent treatise by Marec[1]. As to the fixed-time case, the Lambert problem itself is difficult enough to be dealt with, owing to the transcendental nature of the time equation and its many different forms under different conditions, inherited from the classical Lambert's formulation, not to say the optimization, which amounts to searching for the best solution from the set of isochronous solutions of the infinitely many Lambert problems. In recent years abundant literature is found on the Lambert problem, and good advances have been made by Battin [2] Godal [3], Sun [4 6], and many others for its analysis and solution; but investigations on the fixed-time optimization problem are rather scarce, and despite of the few fine articles by Jezewski[7] Lion [8], Peltier [9], et al. found in the current literature, much analytic and computational work has yet to be done.

The following consists of mainly two parts. In the first part, the Lambertian invariance will be briefly reviewed, and a new invariant, the ratio of two invariable lengths, will be selected to replace the classical Gauss ratio of two areas as the main iterative variable in the time equation. To make use of this new variable, new forms of the time equation will be derived and rearranged so that they can be solved by iteration with fast convergence. In the second part the problem of optimal impulsive transfer under a prescribed transfer

†Professor, Institute of Applied Mathematics.

$\ddagger$ Professor, Department of Aerospace Engineering. time will be treated by the parametrization method. To simplify the problem the model of coplanar circle-tocircle transfer using two impulses will be assumed, and explicit forms of the optimal equations will be derived. The highly transcendental equations thus obtained will then be numerically solved by the iteration procedure developed, and the results graphically displayed and examined. After the simplest case of two terminal orbits in the same direction of motion, and transfer without multi-revolution, is fully investigated, the case of two orbits in counter-directions of motion as well as the long duration case with possible multi-revolution will be briefly treated. Finally, the study will be extended from 2-impulse to 3-impulse transfer, and the variety of fixed time optima under different combinations of radius ratio and transfer time will be reviewed and summarized. It is hoped that the findings from this preliminary study may serve to supplement the existing literature on this difficult problem, and help to pave the way for further investigations.

\section{LAMBERTIAN INVARIANTS}

Consider a Keplerian trajectory between two terminal points $Q_{1}$ and $Q_{2}$ with position vectors $\mathbf{r}_{1}$ and $\mathbf{r}_{2}$ from the field center 0 . The well-known Lambert theorem concerning the time of flight along the trajectory may be written symbolically:

$$
\Delta t=t_{2}-t_{1}=f\left(r_{1}+r_{2}, c, a\right),
$$

where $c$ is the chord length $Q_{1} Q_{2}$, and $a$ is the semimajor axis of the elliptic trajectory $(a>0)$, or the semitransversal axis of the hyperbolic trajectory $(a<0)$, and $a \rightarrow \infty$ if the trajectory is parabolic. Then by Lambertian 
invariant we mean any parameter $X$ which is a function of $r_{1}+r_{2}, c$ and $a$, i.e.

$$
X=X\left(r_{1}+r_{2}, c, a\right),
$$

where any one of $r_{1}+r_{2}, c$ or $a$ may of course be absent. If $a$ is absent, then $X$ is said to be a geometric Lambertian invariant.

Of the many Lambertian invariants found so far, the one of direct concern here is the quantity $r_{p k}$ which is the distance from the field center to the trajectory point $Q_{p k}, \dagger$ where the velocity vector is parallel to the chordline $Q_{1} Q_{2}$ (Fig. 1). This Lambertian invariant, discovered by Battin [2] from Levine's Theorem [10], has also been found by Sun[4] from Hamilton's isochronous hodograph [3], but it was Battin who made a good use of it in his symmetric transformation of the Lambert problem. Directly associated with this invariant, Vinh [11] further shows that the distance $\rho$ from the field center 0 to the point $D$ where the vector $r_{p k}$ intersects the chord $Q_{1} Q_{2}$ (Fig. 1), is also an invariant, since

$$
\rho=\sqrt{r_{1} r_{2}} \cos \frac{\psi_{R}}{2}= \pm \frac{1}{2} \sqrt{\left(r_{1}+r_{2}+c\right)\left(r_{1}+r_{2}-c\right)},
$$

where $\psi_{R}$ is the range angle, which is related to the central angle $\psi$ by:

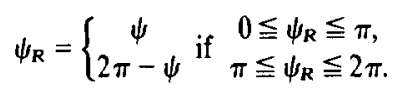

Equation (3) shows that $\rho$ is a geometric invariant, independent of $a$; and:

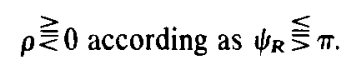

For a fixed base triangle $0 Q_{1} Q_{2}$, the fixed length $|\rho|$ implies that $D$ is a fixed point, and so is the direction of $\mathbf{r}_{p k}$. It follows that all two-terminal trajectories through the same two terminal points and moving in the same direction around the field center will have their peak points lying on a fixed straight line. This deduction confirms the previous findings by Sun[12] through the

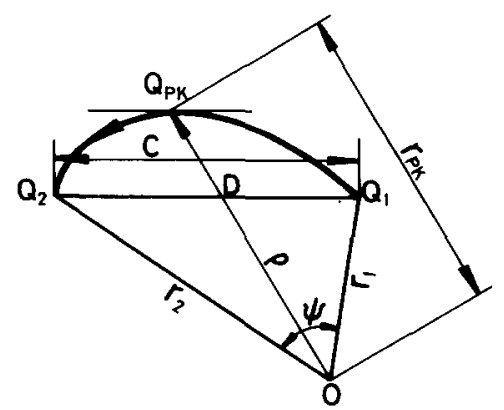

Fig. 1. Lambertian invariant lengths.

tCalled by Levine and Battin the normal point [10, 2]; and by Sun the peak point [4]. hodograph analysis, and relevant formulas are found in the same paper. However, it should be noted that the direction of $\mathbf{r}_{p k}$ is an invariant of the 2-terminal trajectory family over a fixed base triangle, but not a Lambertian invariant.

To facilitate the subsequent development we introduce the invariant ratio:

$$
\Lambda=\left(r_{1}+r_{2}+2 \rho\right) / 4 r_{p k}
$$

It can be shown that $\Lambda$ is related to the two basic Lambertian invariants $\alpha$ and $\beta$ by:

$$
\Lambda=\left\{\begin{array}{l}
\cos ^{2} \frac{1}{4}(\alpha-\beta)=\cos ^{2} \frac{g}{2}, \\
\cosh ^{2} \frac{1}{4}(\alpha-\beta)=\cosh ^{2} \frac{g}{2},
\end{array}\right.
$$

where $\alpha$ and $\beta$ are given by:

$$
\frac{r_{1}+r_{2}+1}{4|a|}=\left\{\begin{array}{l}
\sin ^{2} \frac{\alpha}{2} \\
\sinh ^{2} \frac{\alpha}{2}
\end{array} \frac{r_{1}+r_{2}-1}{4|a|}=\left\{\begin{array}{l}
\sin ^{2} \frac{\beta}{2} \\
\sinh ^{2} \frac{\beta}{2}
\end{array}\right.\right.
$$

and for convenience we have put

$$
g=\frac{1}{2}(\alpha-\beta)
$$

The upper expressions in eqns (6) and (7) are for the elliptic trajectory, while the lower ones, for the hyperbolic trajectory. Such a convention will be followed throughout this paper unless otherwise indicated. It will be seen later that this invariant ratio $\Lambda$ will be the main iterative variable to be used in the solution of the Lamber problem. Besides, the ratio

$$
\epsilon=2 \rho /\left(r_{1}+r_{2}\right)
$$

will also be employed in the following development. Obviously, $\epsilon$, like $\rho$, is also a Lambertian geometric invariant, which is related to the range angle $\psi_{R}$ by

$$
\epsilon=\frac{2 \sqrt{r_{1} r_{2}}}{r_{1}+r_{2}} \cos \frac{\psi_{\mathrm{R}}}{2} .
$$

Hence $\epsilon$ always agrees in sign with $\rho$, and $|\epsilon| \leqq 1$. In the limiting case of $|\epsilon|=1$, we have $r_{1}=r_{2}$, the trajectory will be rectilinear and $\epsilon=+1$, or -1 , according as $\psi_{R}=0$, or $2 \pi$.

\section{SOLUTION OF THE LAMBERT PROBLEM}

By introducing the normalized time of flight

$$
K=\Delta t \frac{\sqrt{\mu}}{2 \tau}\left(\frac{2}{r_{1}+r_{2}}\right)^{3 / 2}
$$

the classical form of the Lambert time equation in the elliptic case takes on the form:

$$
\pi K=\left(\frac{2 a}{r_{1}+r_{2}}\right)^{3 / 2}(g-\cos \gamma \sin g)
$$


where

$$
\gamma=\frac{1}{2}(\alpha+\beta),
$$

and $g$ is defined by Eqn (8). By using Battin's symmetric transformation [2] it can be shown that:

$$
\begin{gathered}
\cos \gamma=(\cos g-\epsilon) / G, \\
\frac{r_{1}+r_{2}}{2 a}=\sin ^{2} g / G,
\end{gathered}
$$

where, $\epsilon$ is defined by Eqn (9), and for convenience we have put

$$
G=1-\epsilon \cos g .
$$

Substituting Eqns (14E) and (15E) into Eqn (12E) yields the elliptic time equation:

$$
\pi K=G^{1 / 2}\left\{\frac{2 g-\sin 2 g}{2 \sin ^{3} g} G+\epsilon\right\} .
$$

In analogous manner the corresponding hyperbolic time equation is found to be

$$
\pi K=G^{1 / 2}\left\{\frac{\sinh 2 g-2 g}{2 \sinh ^{3} g} G+\epsilon\right\},
$$

where $g$ and $\epsilon$ are again defined by Equs (8) and (9) respectively, and

$$
G=1-\epsilon \cosh g .
$$

For two fixed terminal points and a specified $\Delta t$, both $\epsilon$ and $K$ are known, and the single unknown in Eqn (17E or $17 \mathrm{H}$ ) is the variable $g$. These are the basic forms of the time equation to be used for the Lambert problem instead of the classical Gauss equation.

To facilitate the numerical solution we employ the invariant ratio $\Lambda$ defined by Eqn (5), and use relations $(6 \mathrm{E}, \mathrm{H})$ to re-write Eqns $(17 \mathrm{E}, \mathrm{H})$ in the iterative form $\dagger$

$$
\Lambda_{i+1}^{3 / 2}=\frac{G_{i}^{3 / 2}}{2 \pi K}\left(L_{i}+\frac{2 \epsilon}{G_{i}} \Lambda_{i}^{3 / 2}\right)
$$

where

$$
L=\left\{\begin{array}{c}
(2 g-\sin 2 g) /\left(2 \sin \frac{g}{2}\right)^{3} \\
(\sinh 2 g-2 g) /\left(2 \sinh \frac{g}{2}\right)^{3}
\end{array}\right.
$$

and $i$ indicates the step of iteration $(i=0,1,2, \ldots)$. By using $\Lambda$ as the iterative variable, the iteration is found to converge rapidly for

$$
\epsilon<0.2(2 \pi K+1) \text {. }
$$

†A form similar to Eqn (17E) was found by Godal[3] for the elliptic case, but no form for the hyperbolic case, nor the iterative form like Eqn (18).
When this inequality is not satisfied, then to assure rapid convergence the following equation is to be used instead of Eqn (18):

$$
\begin{aligned}
G_{i+1}^{1 / 2}=\left(2 M_{i}\right)^{-1 / 3} & \left\{\left[\left(4 \pi^{2} K^{2}+\frac{32 \epsilon^{3}}{27 M_{i}}\right)^{1 / 2}+2 \pi K\right]^{1 / 3}\right. \\
- & \left\{\left[\left(4 \pi^{2} K^{2}+\frac{32 \epsilon^{3}}{27 M_{i}}\right)^{1 / 2}-2 \pi K\right]^{1 / 3},\right.
\end{aligned}
$$

where we have put:

$$
M=\left\{\begin{array}{c}
(2 g-\sin 2 g) / \sin ^{3} g, \\
(\sinh 2 g-2 g) / \sinh ^{3} g .
\end{array}\right.
$$

A detailed analysis on the convergence of the iteration is found in Vinh [11], and the hypergeometric expansion of the function $M$ and techniques for its evaluation is available in Battin [2].

In the parabolic limit, $g \rightarrow 0$, both equations $(17 \mathrm{E}, \mathrm{H})$ yield the nondimensional time of parabolic transfer:

$$
K_{\mathrm{p}}=\frac{1}{3 \pi}(2+\epsilon)(1-\epsilon)^{1 / 2} .
$$

However, when $g$ is near zero, the iterative routine using Eqn (21) becomes highly sensitive to error. To remedy this situation, let

$$
\zeta=1-\Lambda=\left\{\begin{array}{c}
\sin ^{2} \frac{g}{2} \\
-\sinh ^{2} \frac{g}{2}
\end{array}\right.
$$

and

$$
\delta=10 \pi\left(K-K_{p}\right) ;
$$

then by series expansion we found:

$$
\zeta=\sum_{j=1}^{\infty} a_{j} \delta^{j} .
$$

The first few coefficients in Eqn (25), are found as follows:

$$
\begin{aligned}
a_{1}= & \frac{(1-\epsilon)^{1 / 2}}{8 A} \\
a_{2}= & -\frac{1}{56 A^{3}}\left[1-\frac{3}{4}\left(\frac{\epsilon}{2}\right)-\frac{37}{8}\left(\frac{\epsilon}{2}\right)^{2}-\frac{1}{8}\left(\frac{\epsilon}{2}\right)^{3}\right] \\
a_{3}= & \frac{(1-\epsilon)^{1 / 2}}{441 A^{5}}\left[\frac{37}{32}+\frac{11}{16}\left(\frac{\epsilon}{2}\right)-\frac{2615}{256}\left(\frac{\epsilon}{2}\right)^{2}-\frac{2117}{128}\left(\frac{\epsilon}{2}\right)^{3}\right. \\
& \left.-\frac{197}{32}\left(\frac{\epsilon}{2}\right)^{4}-\frac{85}{32}\left(\frac{\epsilon}{2}\right)^{5}\right] \\
a_{4}= & -\frac{5}{1176 A}\left[\frac{169}{1848}-\frac{1}{924}\left(\frac{\epsilon}{2}\right)-\frac{2617}{1760}\left(\frac{\epsilon}{2}\right)^{2}\right. \\
& -\frac{3619}{1760}\left(\frac{\epsilon}{2}\right)^{3}+\frac{4967}{1536}\left(\frac{\epsilon}{2}\right)^{4}+\frac{140123}{16896}\left(\frac{\epsilon}{2}\right)^{5} \\
& \left.+\frac{60583}{8448}\left(\frac{\epsilon}{2}\right)^{6}+\frac{112391}{24640}\left(\frac{\epsilon}{2}\right)^{7}+\frac{69341}{73920}\left(\frac{\epsilon}{2}\right)^{8}\right]
\end{aligned}
$$


where we have defined

$$
A=1+\left(\frac{\epsilon}{2}\right)-\left(\frac{\epsilon}{2}\right)^{2}
$$

The details are again found in Vinh[11], and it is shown there that the series solution is quite accurate for near parabolic transfer.

\section{THE OPTIMAL FIXED-TLME, IMPULSIVE TRANSFER BETWEEN CIRCULAR ORBITS: BASIC TREATMENT}

\subsection{Introductory remarks}

Consider the transfer between two circular orbits $C_{1}$ and $C_{2}$ of radii $r_{1}$ and $r_{2}$ respectively in the same plane, and let:

$$
n=r_{2} / r_{1}
$$

For definiteness we assume $r_{2}>r_{1}$, or $n>1$, and consider the transfer from the inner orbit to the outer. This is actually no restriction, since the time of transfer will not be affected by interchanging $r_{1}$ and $r_{2}$, and the solution will be essentially valid for the transfer from the outer to the inner. However, the following two cases will be distinguished:

(1) uni-rotating: $C_{1}$ and $C_{2}$ in the same rotational direction around the field center;

(2) counter-rotating: $C_{1}$ and $C_{2}$ in opposite directions of rotation.

\subsection{Formulation of the problem}

With reference to Figs. 2 and 3 we define the normalized terminal velocity $\nu_{i}$ and velocity impulse $f_{i}$ by:

$$
\begin{gathered}
\nu_{i}=\mathbf{V}_{i} / \sqrt{\frac{\mu}{r_{i}}}, \\
(i=1,2) \\
f_{i}=\left|\Delta \mathbf{V}_{i}\right| / \sqrt{\frac{\mu}{r_{1}}},
\end{gathered}
$$
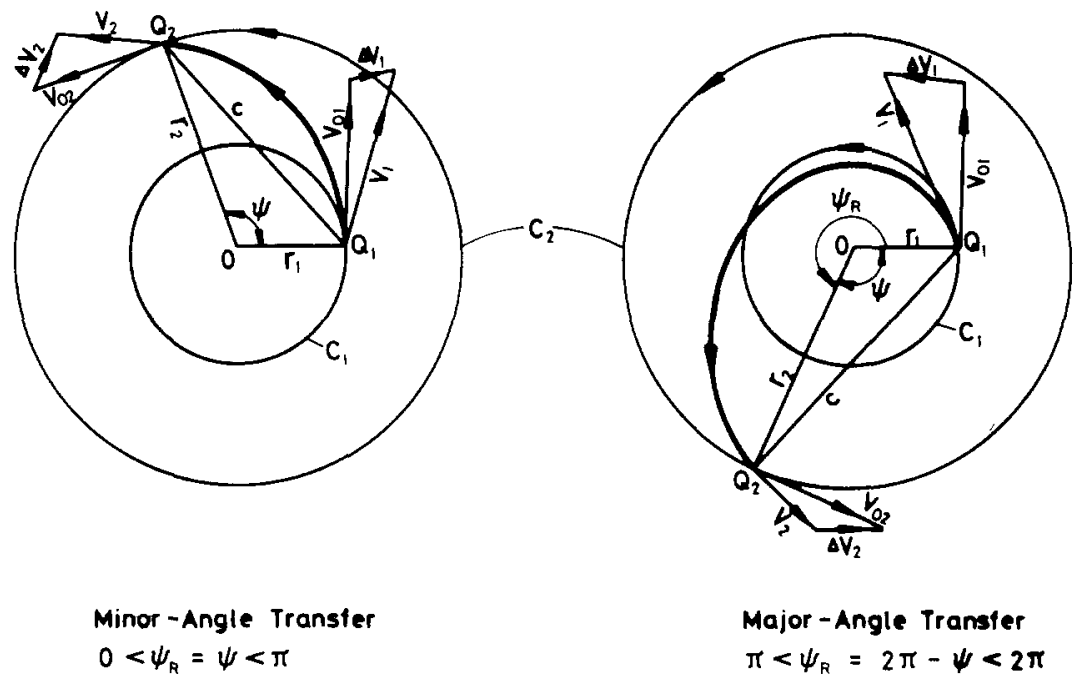

Fig. 2. Transfer between circular orbits: uni-rotating case.

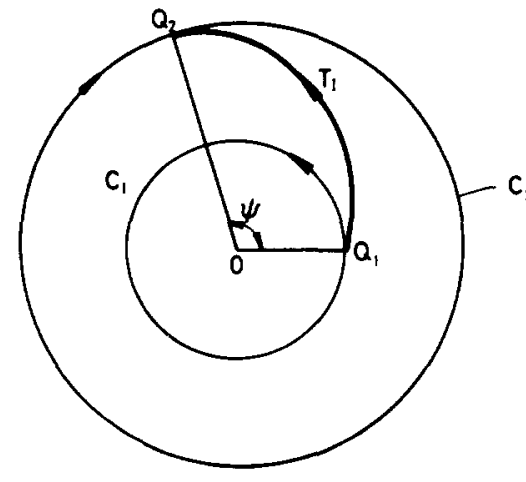

A. Transfer in Direction of $C_{1}$

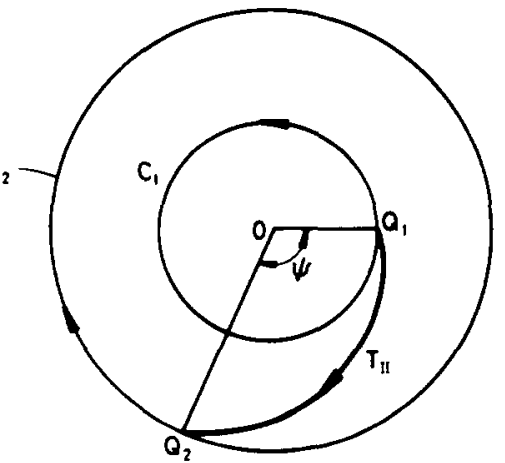

B. Transter in Direction of $C_{2}$

Fig. 3. Transfer between circular orbits: counter-rotating case. 
where:

$$
\begin{gathered}
f_{1}=\left|\Delta \nu_{1}\right|=\left|\nu_{1}-\nu_{01}\right|, \\
f_{2}=\left|\Delta \nu_{2}\right| / \sqrt{n}=\left|\nu_{2}-\nu_{02}\right| / \sqrt{n} .
\end{gathered}
$$

The subscript 0 here refers to the terminal condition before applying the velocity impulse. The normalized characteristic speed to be minimized is then:

$$
f=f_{1}+f_{2}
$$

under the constraint of fixed $\Delta t$ or $K$ defined by eqn (11).

To develop eqns (31) further and to avoid confusion we take the transversal- or $\theta$-component of velocity in the direction of $C_{1}$ as positive for both cases of unirotating and counter-rotating; and we will take up the uni-rotating case first. In this case, both $C_{1}$ and $C_{2}$ and the transfer trajectory all agree in the direction of motion, hence:

$$
\left(\nu_{\theta}\right)_{01}=\left(\nu_{\theta}\right)_{02}=+1 ; \nu_{\theta 1}>0, \nu_{\theta 2}>0 ;
$$

and following eqns $(31-1,2)$ we have:

$$
\begin{gathered}
f_{1}^{2}=\nu_{1}^{2}-2 \nu_{\theta 1}+1, \\
f_{2}{ }^{2}=\left(\nu_{2}^{2}-2 \nu_{\theta 2}+1\right) / n,
\end{gathered}
$$

which, in terms of $\epsilon$ and $g$, may be expressed as:

$$
\begin{aligned}
& f_{1}{ }^{2}=3-\frac{2}{n+1} \phi-2 \nu_{\theta 1}, \\
& f_{2}^{2}=\frac{3}{n}-\frac{2}{n+1} \phi-\frac{2 \nu_{\theta 1}}{n^{3 / 2}},
\end{aligned}
$$

where

$$
\begin{gathered}
\nu_{\theta 1}=\left[\frac{4 n-(n+1)^{2} \epsilon^{2}}{2(n+1) G}\right]^{1 / 2}, \\
\phi=\left\{\begin{array}{c}
\sin ^{2} g / G, \\
-\sinh ^{2} g / G,
\end{array}\right.
\end{gathered}
$$

and $G$ is defined by eqns (15). It should be noted at this point that, in view of eqn (10), $\epsilon$ is bounded as follows:

$$
|\epsilon| \leqq \frac{2 \sqrt{n}}{n+1}
$$

Following eqns $(17 \mathrm{E}, \mathrm{H})$ derived in Section 2, the time constraint may be written as

$$
\pi K=\Phi(\epsilon, g)
$$

with $\Phi$ defined by

$$
\Phi(\epsilon, g)=\left\{\begin{array}{l}
G^{3 / 2}(2 g-\sin 2 g) / 2 \sin ^{3} g+G^{1 / 2} \epsilon \\
G^{3 / 2}(\sinh 2 g-2 g) / 2 \sinh ^{3} g+G^{1 / 2} \epsilon .
\end{array}\right.
$$

\subsection{The optimal solution}

To minimize $f$ under the constraint (39) we employ the Lagrange multiplier $\lambda$ and introduce the augmented performance index :

$$
\bar{f}=f+\lambda(\Phi-\pi K)
$$

The optimal condition may then be written as:

$$
\begin{gathered}
\frac{\partial f}{\partial \epsilon}+\lambda \frac{\partial \Phi}{\partial \epsilon}=0, \\
\frac{\partial f}{\partial g}+\lambda \frac{\partial \Phi}{\partial g}=0, \\
\Phi(\epsilon, g)-\pi K=0 .
\end{gathered}
$$

Eliminating $\lambda$ from eqns $(42,43)$ results in the vanishing of the Jacobian

$$
\frac{\partial(f, \Phi)}{\partial(\epsilon, g)}=0
$$

which, after carrying out the differentiation and simplifying, yields the optimal condition:

$$
\epsilon\left\{\frac{(2 n)^{3}\left[4 n-(n-1)^{2} \epsilon^{2}\right.}{n+1}\right\}^{1 / 2} \frac{f_{1}+f_{2}}{f_{1}+n^{3 / 2} f_{2}}=\Psi(\epsilon, g, n),
$$

where:

$$
\begin{aligned}
& \Psi(\epsilon, g, n)= \\
& \left\{\begin{array}{l}
\left\{(n+1)^{2} \epsilon \chi-\left[4 n-(n+1)^{2} \epsilon^{2}\right] \omega\right\} / \sin ^{2} g, \\
-\left\{(n+1)^{2} \epsilon \chi-\left[4 n-(n+1)^{2} \epsilon^{2}\right] \omega\right\} / \sinh ^{2} g,
\end{array}\right.
\end{aligned}
$$

and

$$
\begin{gathered}
\chi=\left\{\begin{array}{c}
2\left(1-\epsilon^{2}+G\right) G^{1 / 2}+3 \pi K[\epsilon-(1+G) \cos g], \\
2\left(1-\epsilon^{2}+G\right) G^{1 / 2}+3 \pi K[\epsilon-(1+G) \cosh g]
\end{array}\right. \\
\omega=\left\{\begin{array}{c}
(2 \cos g+\epsilon) G^{1 / 2} \times 3 \pi K \cos ^{2} g \\
(2 \cosh g+\epsilon) G^{1 / 2}-3 \pi K \cosh ^{2} g
\end{array}\right.
\end{gathered}
$$

To apply the optimal equation (45) it is necessary to predetermine whether the optimal solution is to be elliptic or hyperbolic. To do this, we let $g \rightarrow 0$, and found that both eqns $(46 \mathrm{E}, \mathrm{H})$ approach the same parabolic limit:

$$
\begin{aligned}
& \psi_{p}=\frac{1}{5}(1-\epsilon)^{1 / 2}\left\{(n+1)^{2} \epsilon\left(4+2 \epsilon-\epsilon^{2}\right)\right. \\
&\left.-(2+3 \epsilon)\left[4 n-(n+1)^{2} \epsilon^{2}\right]\right\} .
\end{aligned}
$$

Meantime, with $g=0$, combining eqns (35) with (36), (37) gives

$$
f_{1 p}=\left\{3-2\left(\frac{4 n-(n+1)^{2} \epsilon^{2}}{2(n+1)(1-\epsilon)}\right)^{1 / 2}\right\}^{1 / 2},
$$




$$
f_{2 p}=\left\{\frac{3}{n}-\frac{2}{n^{3 / 2}}\left(\frac{4 n-(n+1)^{2} \epsilon^{2}}{2(n+1)(1-\epsilon)}\right)^{1 / 2}\right\}^{1 / 2} .
$$

Substituting eqns (35P), (46P) into eqn (45) yields an equation

$$
F(\epsilon, n)=0 \text {. }
$$

With $n$ given, and $\epsilon$ restricted to $|\epsilon| \leqq 2 \sqrt{n} /(n+1)$, eqn (49) gives the critical value of $\epsilon_{p}^{*}$, and the corresponding critical time of flight is then:

$$
K_{p}^{*}=\frac{1}{3 \pi}\left(2+\epsilon_{p}^{*}\right)\left(1-\epsilon_{p}^{*}\right)^{1 / 2},
$$

Consequently, with the specified values of $K$ and $n$ in an orbital transfer problem we have the criterion:

$$
\left.K \geqq K_{p}^{*} \Rightarrow \begin{array}{l}
\text { elliptic } \\
\text { parabolic } \\
\text { hyperbolic }
\end{array}\right\} \text { optimum. }
$$

The critical boundary separating the regions of elliptic and hyperbolic optimum in the $n, K$-plane is shown in Fig. 4. On the boundary, $K^{*}$ is seen to be monotonically increasing with $n$, with

$$
\lim _{n \rightarrow \infty} K_{p}^{*}=2 / 3 \pi
$$

which can be obtained directly from eqn (50), since $\epsilon_{p}^{*} \rightarrow 0$ as $n \rightarrow \infty$. With the nature of the optimal solution thus predetermined, the appropriate form of eqn (45) as well as that of eqn (40) may now be chosen, and solution of this pair of equations will give the optimal values of $\epsilon$ and $g$ for minimum $f$.

A typical graph illustrating the numerical solutions for the case $n=2$ at several values of $K$ is shown in Fig. 5 . From such graphical display we observe that

(1) At fixed distance ratio $n$, for each value of $K$ prescribed there is an absolute minimum $f$, which occurs at

$$
\psi \lesseqgtr 180^{\circ} \text { according as } K \gtreqless 0.5 \text {. }
$$

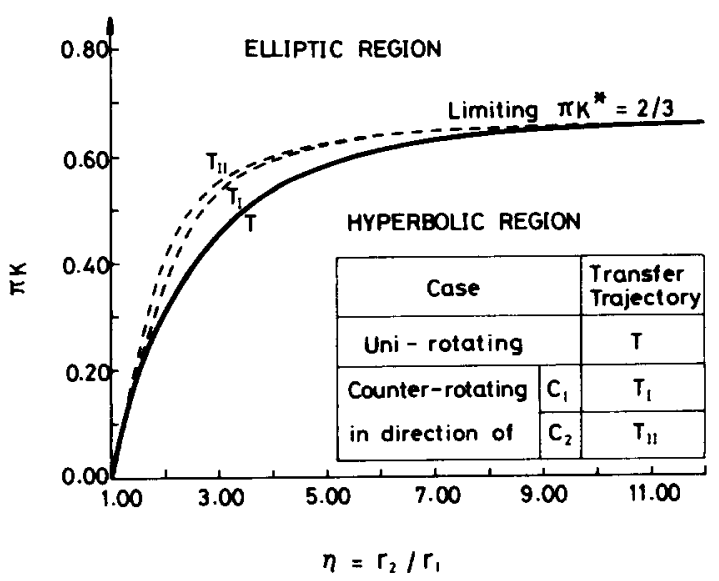

Fig. 4. Boundary of critical optimum.
In particular, at $K=0.5, \psi=180^{\circ}, f$ reaches its overall minimum and it can be shown that this transfer trajectory is the Hohmann tangential ellipse.

(2) At fixed $n$, the higher the value of $K$, the greater is the optimal range angle $\psi_{R^{* *}}$ for minimum $f$. Figure 5 shows that as $K \rightarrow \infty$ at $n=2$, the limiting value of $\psi_{R^{* *}}$ is $270^{\circ}$. In general it can be shown that

$$
\lim _{K \rightarrow \infty} \psi_{R^{* *}}=2 \cos ^{-1}\left(-\frac{1}{\sqrt{n}}\right) \quad(n \geqq 1) .
$$

(3) For short duration the optimum may be hyperbolic even the time prescribed allows elliptic transfer. For example, at $n=2$, hyperbolic transfer is optimal for $K<K_{p}^{*}=0.1175$ (see Fig. 5).

The above observations show that whenever $\mathrm{K} \neq 0.5$, the optimum is less economical than the Hohmann. However, if the transfer time prescribed is $K>0.5$, we can still use the Hohmann transfer by letting the space vehicle stay on the original orbit for a sufficient time $\Delta t_{D}$ before the impulsive transfer is started, so that the effective time parameter is:

$$
K-K_{\mathrm{D}}=0.5,
$$

where

$$
K_{D}=\Delta t_{D} \frac{\sqrt{\mu}}{2 \pi}\left(\frac{2}{r_{1}+r_{2}}\right)^{3 / 2}
$$

is the time parameter corresponding to $\Delta t_{D}$. The required delay period $\Delta t_{D}$ is determined from eqns (53), (54), and the effective time of transfer is then $\Delta t-\Delta t_{D}$. Thus when such a delay is allowed, the optimum solution will always be Hohmann for any $K>0.5$. If this is not allowed, then the optimal elliptic solution can only be improved by resorting to multi-revolution, as presented in the next section.

\section{LONG DURATION TRANSFER WITH MULTI-REVOLUTION}

If $N$ is the number of complete revolutions to be made in addition to the net arc connecting $Q_{1}$ and $Q_{2}$, the time equation (17E) needs be simply modified as follows:

$$
\pi K=G^{1 / 2}\left\{\frac{2 g+2 \pi N-\sin 2 g}{2 \sin ^{3} g} G+\epsilon\right\}
$$

The convergence character in solving this equation is essentially the same as solving eqn (17E), and the optimal equation (45) still stands with eqn (40E) replaced by eqn (55) in the defining equation of $\Phi$.

To determine the maximum possible value of $N$ for a given $K$, it is important to note that there exists a minimum time of transfer [5] for each $N>0$. A direct differentation of eqn (55) gives

$$
K_{\min }=\frac{2}{3 \pi} G^{1 / 2} \frac{2-\epsilon \cos g-\epsilon^{2}}{2 \cos g-\epsilon \cos ^{2} g-\epsilon}
$$




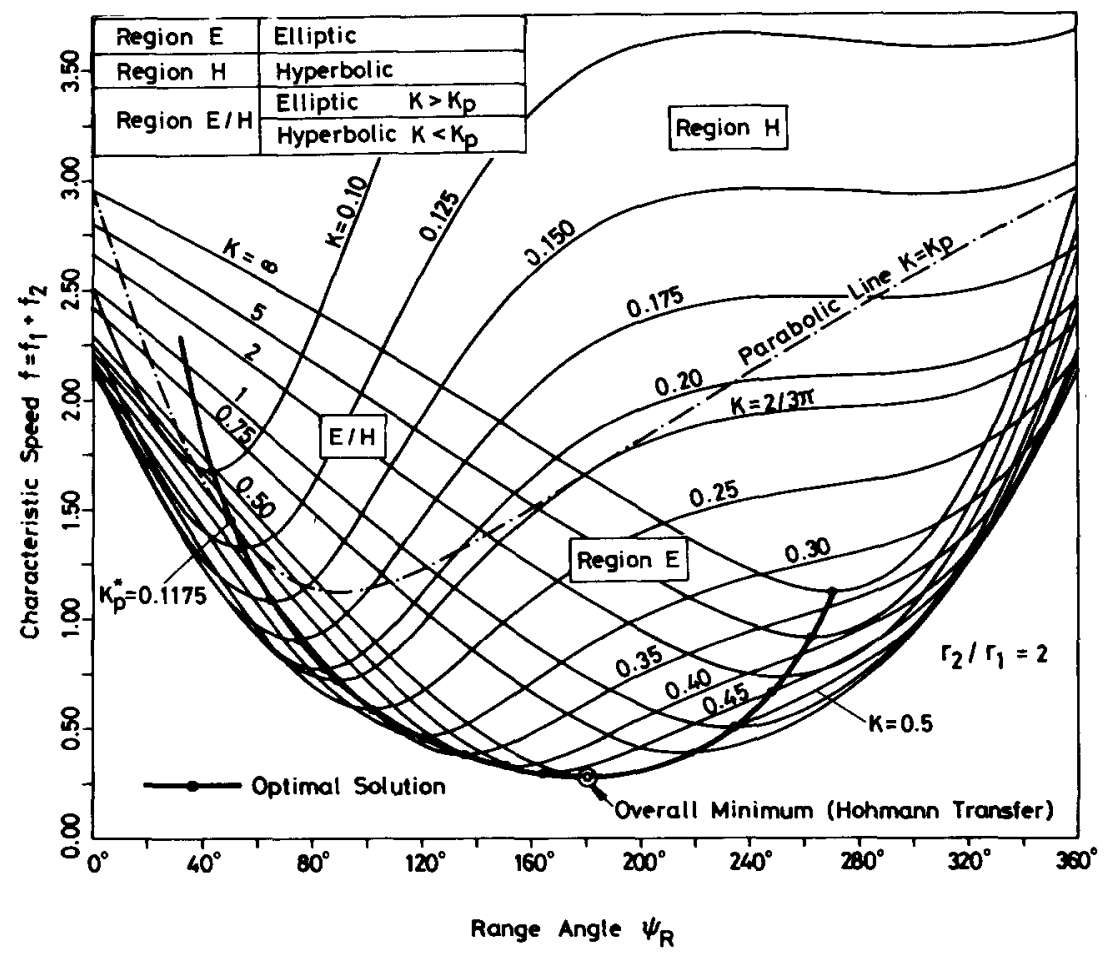

Fig. 5. Variation of characteristic speed with range angle, and the optimal solutions $\left(r_{2} / r_{1}=2\right)$.

where $g$ satisfies the equation:

$$
2 \pi N+2 g-\sin 2 g=\frac{2 \sin ^{3} g\left(4 G^{2}+\epsilon^{2} \sin ^{2} g\right)}{3 G\left(2 \cos g-\epsilon \cos ^{2} g-\epsilon\right)} .
$$

In the particular case of $\epsilon=0$, eqns (56), (57) reduce to

$$
K_{\min }=\frac{2}{3 \pi} \sec g
$$

and

$$
g+N \pi=\frac{1}{3} \tan g\left(2+\cos ^{2} g\right)
$$

an approximate solution of which may be written[5]:

$$
K_{\min } \cong\left(N+\frac{1}{2}\right)\left\{1-\frac{8}{3(2 N+1)^{2} \pi^{2}}\right\},
$$

which shows that $K_{\min }$ is slightly less than $N+0.5$, and tends to this value when $N$ is large. It should be noted that in deriving eqns (56) and (57) we have tacitly assumed that $|\epsilon| \neq 1$. When $\epsilon=+1$, they fail to apply, but it can be easily shown that

$$
g=0, K_{\min }=N / 2 \sqrt{2},
$$

since the net transfer arc now is null, and the minimum time trajectory consists of just $N$ complete rectilinear circuits on a flat ellipse. On the other hand, when $\epsilon=-1$, we found that eqns (56), (57) are still valid, giving:

$$
K_{\min }=\frac{\sqrt{2}}{3 \pi} \sec \frac{g}{2}
$$

and

$$
\frac{1}{2}(g+N \pi)=\frac{1}{3} \tan \frac{g}{2}\left(2+\cos ^{2} \frac{g}{2}\right) .
$$

A close examination of these equations shows that the net transfer arc in this case is not simply a complete flat ellipse, so that $K_{\min }=(N+1) / 2 \sqrt{2}$ as we might expect, but a rectilinear segment. An appropriate solution [5] for this case is:

$$
K_{\min } \cong \frac{N+1}{2 \sqrt{2}}\left\{1-\frac{8}{3(N+1)^{2} \pi^{2}}\right\},
$$

which shows that $K_{\min }$ does tend to the value $(N+$ 1)/2 $\sqrt{2}$ at large $N$.

With the $K_{\min }$ associated with a given $N$ thus determined, a careful analysis enables one to infer the following:

(1) The maximum possible number of complete revolutions is $N_{\max }=m$, if:

$$
m \leqq K\left(1+\sigma^{2}\right)^{3 / 2}<m+1,
$$

where $m$ is an integer $\geqq 0$, and $\sigma$ is a parameter related to $\epsilon$ by:

$$
\epsilon=\frac{2 \sigma}{1+\sigma^{2}}, \text { or } \sigma=\frac{1}{\epsilon}\left\{1-\sqrt{1-\epsilon^{2}}\right\} \text {. }
$$


(2) With $m$ found for a specified $K$, the time equation (55) with $N=m$ will give two distinct solutions, a unique solution, or no solution according as $\boldsymbol{K}$ is greater than, equal to, or less than $K_{\text {min }}$.

(3) If $N_{\max }=m$, then there exist two distinct solutions for each value of $N=m-1, m-2, \ldots, 1$; and one solution for $N=0$. A detailed analysis on the number and nature of the multi-revolution solutions is given in [5]. It should be mentioned that the parameter $\sigma$ defined here always agree in sign with $\epsilon$, and that:

$$
\epsilon=\left\{\begin{array}{r}
+1 \\
0 \\
-1
\end{array} \quad \Leftrightarrow \sigma=\left\{\begin{array}{r}
+1 \\
0 \\
-1
\end{array}\right.\right.
$$

Furthermore, it is related to the Lambertian invariants $r_{1}+r_{2}, c$, and $\rho$ as follows:

$$
\sigma^{2}=\left(r_{1}+r_{2}-c\right) /\left(r_{1}+r_{2}+c\right)=\left[2 \rho /\left(r_{1}+r_{2}+c\right)\right]^{2} .
$$

To facilitate the solution the lines of minimum transfer time are plotted in the $K, \sigma$-plane as shown in Fig. 6 , from which the maximum number of complete revolutions that can be realized may be readily assessed and

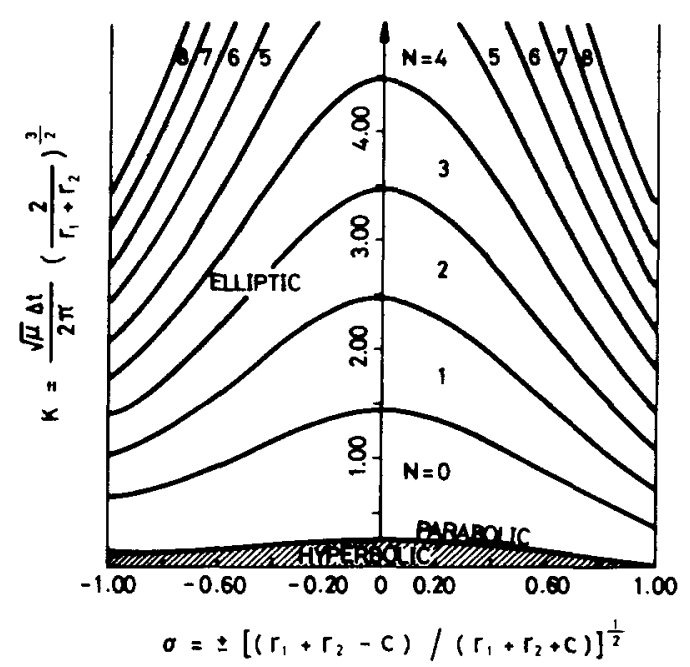

Fig. 6. The minimum time boundaries and the regions for multirevolution transfer. the number of solutions predetermined once the time parameter $K$ is specified, in accordance with items 2 and 3 stated above.

With the possible number of complete revolutions determined, the optimization problem can be handled in the same manner as in the non-multi-revolution case by solving the optimal equation (45) in elliptic form, together with the time equation now replaced by its modified form (55). However, the following complications are to be noted:

(1) For each $N \in\left[1, N_{\max }\right)$ there are two distinct solutions given by the time equation (55), and the same is true for $N=N_{\max }$ and $K>K_{\min }$. Consequently, two optimal solutions will be provided by eqns (45) and (55) to each $N$ assigned, and they are to be compared.

(2) The overall optimization will consist of selecting the one giving the lowest $f$ from the $m+1$ sets of optimal solutions corresponding to $N=0,1, \ldots$, $N_{\text {max }}(=m)$.

Consequently for fixed $n$ and $K$, the variable $N$ is yet to be optimized in addition to $\epsilon$ and $g$. Thus the problem is much more involved; and further analysis is needed for its treatment, and efficient iteration routine has to be developed. However, despite of these complications the following simple rule can be easily verified:

If $K=q / 2, q$ being any positive odd integer, then the optimal solution for the multi-revolution case will be Hohmann, with the optimal number of revolutions:

$$
N_{* *}=(q-1) / 2 \text {, }
$$

where the subscript ${ }^{* *}$ indicates 2 -impulse optimum. For example:

$$
\begin{array}{lll}
q=1 & K=0.5 & N_{* *}=0, \\
q=3 & K=1.5 & N_{* *}=1, \\
q=5 & K=2.5 & N_{* *}=2 .
\end{array}
$$

The optimal trajectory will all be identical-the Hohmann transfer ellipse. This is the best we can expect from multi-revolution; and whenever $K \neq q / 2$, the optimal solution will give a higher $f$. For illustration purpose a few numerical results are summarized in Table 1. These figures show that in the long duration of transfer the characteristic speed can be prohibitively high if

Table 1. Optimal solution for long duration transfer with multi-revolution (at $r_{1} / r_{1}=2$ )

\begin{tabular}{|c|c|l|c|l|l|l|}
\hline \multirow{2}{*}{$\mathrm{N}$} & \multicolumn{2}{|c|}{$\mathrm{K}=3.25$} & \multicolumn{2}{c|}{$\mathrm{K}=3.5$} & \multicolumn{2}{c|}{$\mathrm{K}=3.75$} \\
\cline { 2 - 7 } & $\mathrm{f}_{\star \star}$ & $\psi_{\star \star}$ & $f_{\star \star}$ & $\psi_{\star \star}$ & $\mathrm{f}_{\star \star}$ & $\psi_{\star \star}$ \\
\hline 0 & 0.83990 & $258.366^{\circ}$ & 0.85386 & $259.086^{\circ}$ & 0.86624 & $259.710^{\circ}$ \\
1 & 0.64483 & $245.4^{\circ}$ & 0.67041 & $247.4^{\circ}$ & 0.69285 & $249.1^{\circ}$ \\
2 & 0.44610 & $224.1^{\circ}$ & 0.48728 & $229.4^{\circ}$ & 0.52256 & $233.6^{\circ}$ \\
3 & $\underline{0.43807}$ & $124.6^{\circ}$ & $\underline{0.28446}$ & $180^{\circ}$ & $\underline{0.32930}$ & $\frac{202.8^{\circ}}{4.61 .5^{\circ}}$ \\
4 & 0.95394 & $64^{\circ}$ & 0.80516 & $77.2^{\circ}$ & 0.67090 & $91.1^{\circ}$ \\
5 & 1.46976 & $31.1^{\circ}$ & 1.25467 & $43.3^{\circ}$ & 1.08905 & $54.1^{\circ}$ \\
6 & & & 1.98287 & $6.4^{\circ}$ & 1.56714 & $26.15^{\circ}$ \\
\hline
\end{tabular}

Note: The figures underlined are the overall optimal at the optimal value of $N_{k *}=3$. 
multi-revolution is not applied; but by using the optimal number of complete revolutions, it can be effectively reduced, and the fuel economy is considerably improved. In the most favorable case, $K=q / 2$, even the Hohmann solution can be achieved by taking $N=(q-1) / 2$ as speculated above. A comprehensive presentation of the multi-revolution case is, however, beyond the scope of the present paper.

\section{THE UNE-ROTATING TRANSFER VS COUNTER-ROTATING TRANSFER}

So far we have restricted ourselves to the uni-rotating case. When the counter-rotating case is concerned, the transfer may take either of the two modes: (I) in the direction of $C_{1}$, or (II) in that of $C_{2}$. Let $T$ denote the transfer trajectory in the uni-rotating case, and $T_{1}$ and $T_{\text {II }}$, the transfer trajectories for Modes I and II respectively in the counter-rotating case. By keeping $\nu_{\theta 1}$ positive in the direction of $C_{1}$ for all cases, then the characteristic speeds of the three cases may be written as follows:

$$
\begin{aligned}
& f=f_{1}+f_{2}=\left(3-\frac{2 \phi}{n+1}-2 \nu_{\theta 1}\right)^{1 / 2}+\left(\frac{3}{n}-\frac{2 \phi}{n+1}-\frac{2 \nu_{\theta 1}}{n^{3 / 2}}\right)^{1 / 2}, \\
& f_{1}=f_{\mathrm{II}}+f_{\mathrm{I} 2}=\left(3-\frac{2 \phi}{n+1}-2 \nu_{\theta 1}\right)^{1 / 2} \\
& +\left(\frac{3}{n}-\frac{2 \phi}{n+1}+\frac{2 \nu_{\theta 1}}{n^{3 / 2}}\right)^{1 / 2} \\
& f_{\mathrm{II}}=f_{\mathrm{III}}+f_{\mathrm{II} 2}=\left(3-\frac{2 \phi}{n+1}+2 v_{\theta 1}\right)^{1 / 2} \\
& +\left(\frac{3}{n}-\frac{2 \phi}{n+1}-\frac{2 \nu_{\theta 1}}{n^{3 / 2}}\right)^{1 / 2},
\end{aligned}
$$

where $\nu_{\theta 1}$ is given by eqn (36).

It should be noted that, for a given $\epsilon$, the value of $n$ is restricted to the range $\left[1, n_{\max }\right]$, where

$$
n_{\max }=\left(1+\sqrt{1-\epsilon^{2}}\right)^{2} / \epsilon^{2}
$$

according to the inequality (38); and at $n=n_{\max }$, we have $\nu_{\theta 1}=0$. With this in mind, a comparison of eqns (69) to (71) shows that for the same $\epsilon, k$, and $n$ (hence the same $\phi$ and $\nu_{\theta 1}$ ), we have

$$
f<f_{\mathrm{I}}<f_{\mathrm{II}} \text { for } 1<n<n_{\max }
$$

and at the endpoints:

$$
\begin{gathered}
n=1: \quad f<f_{\mathrm{I}}=f_{\mathrm{II}}, \\
n=n_{\text {max }}: \quad f=f_{\mathrm{I}}=f_{\mathrm{II}} \text { if } \epsilon \neq 0 .
\end{gathered}
$$

Besides, we note the special case of $\epsilon=0\left(180^{\circ}\right.$ transfer $)$,

$$
n_{\max } \rightarrow \infty \quad f=f_{\mathrm{I}}<f_{I I} .
$$

Such a comparison is shown graphically in Fig. 7. The effect of counter-rotating on the critical boundary is

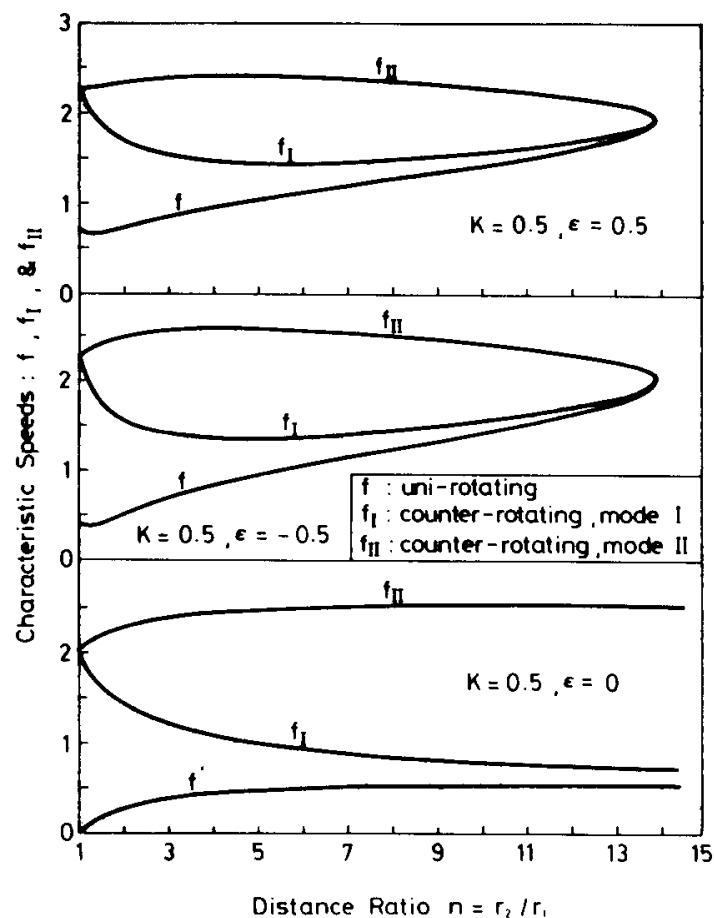

Fig. 7. Variation of characteristic speeds with distance ratio: uni-rotating versus counter-rotating.

shown by the dashed lines in Fig. 4. Denoting

$$
\Delta f=f_{\mathrm{I}}-f ; \quad \Delta f_{\mathrm{I}, \mathrm{II}}=f_{\mathrm{II}}-f_{\mathrm{I}},
$$

an examination of Fig. 7 shows that $\Delta f$ decreases monotonically as $n$ increases for all $|\epsilon|<1$; and $\Delta f_{\mathrm{I}, \text { II }}=0$ at $n=1$, or $n_{\max }$, and reaches its maximum at some intermediate value of $n$ if $\epsilon \neq 0$; but it increases monotonically as $n$ increases if $\epsilon=0$. Furthermore, it is easy to verify from eqns (69) to (71) that at $\epsilon=0$, and when $n \rightarrow \infty$ :

$$
f=f_{\mathrm{I}}=\sqrt{2}-1, \quad f_{\mathrm{II}}=\sqrt{2}+1 ; \quad \Delta f_{\mathrm{I} . \mathrm{II}}=2
$$

for all $K>0$.

Now by keeping $n$ and $K$ constant, and letting the range angle $\psi_{R}$ vary from $0^{\circ}$ to $360^{\circ}$ the variations of $f, f_{I}$ and $f_{I I}$ with $\psi_{R}$ are shown graphically in Fig. 8. An examination of these typical curves shows that, for $n>$ 1 , and $K>0$ :

$$
\begin{aligned}
& f<f_{\mathrm{I}}<f_{\mathrm{II}} \text { for } 0^{\circ}<\psi_{R}<360^{\circ}, \\
& f=f_{\mathrm{I}}=f_{\mathrm{II}} \text { for } \psi_{R}=0 \text {, or } 360^{\circ} .
\end{aligned}
$$

Furthermore, $f$ and $f_{\mathrm{I}}$ each has an absolute minimum at nearly the same $\psi_{R}$ and $\Delta f$ is greatest there; while $f_{\mathrm{II}}$ may have several stationary points on the range $0<\psi_{R}<$ $360^{\circ}$.

Summarizing these observations we may now conclude:

(1) So far as the characteristic speed is concerned, the transfer in the counter-rotating case is more expensive 


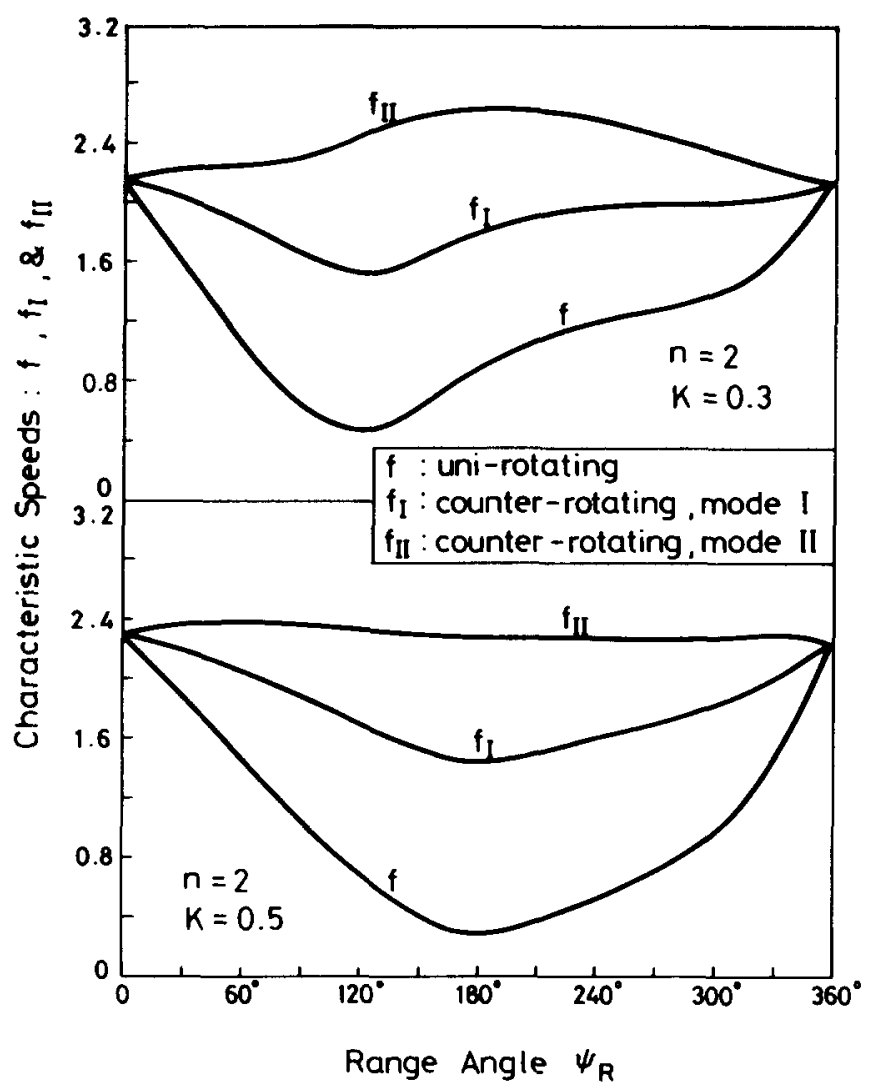

Fig. 8. Variation of characteristic speeds with range angle: uni-rotating versus counter-rotating

than in the uni-rotating case; and among the two modes of transfer for counter-rotating, the transfer in the direction of the outer circle (Mode II) is definitely unfavorable, and should be avoided whenever possible.

(2) Changing from the uni-rotating case to the mode I of the counter-rotating case, the optimal range angle is practically unchanged, but the increase in the characteristic speed is quite significant. For example, Fig. 8 shows that at $n=2$ and $K=0.5: f_{1} \cong 5 f$ at the optimal point (Hohmann).

\section{A GLOBAL SYNTHESIS}

From the previous analysis we see that the overall optimal solution for the fixed-time, 2-impulse transfer between circular orbits is the Hohmann tangential ellipse, and the optimum time parameter is 0.5 . When this is exceeded, the optimal solution will no longer be Hohmann, but the intersecting ellipse, with a higher characteristic speed, but we can still achieve the Hohmann optimum by using the delay period, or in sufficiently long duration transfer we may improve the non-Hohmann solution by using multi-revolution.

However, the 2-impulse Hohmann transfer is optimal only when $n<11.94$, and when this ratio is exceeded, the optimum will be the 3-impulse, biparabolic transfer in the time-free case, according to Hoelker[13] and Marec [4]. In the fixed-time case the biparabolic transfer is not permitted, since the transfer time would be unbounded, and another mode of transfer has to be considered. A simple substitute for the parabolic transfer is Hoelker's 3-impulse bielliptic tangential transfer. Let $n_{c}=r_{c} / r_{1}$, where $r_{\mathrm{c}}$ is the radial distance of the conjunction point, where the two half-ellipses meet, then as shown by Hoelker[13], when $11.94<n<15.58$, in order the bielliptic tangential transfer to be more economical than the Hohmann $n_{c}$ has to exceed some critical value $n_{c}^{*}$ governed by the equation:

$$
\frac{n_{c}-1}{\sqrt{n_{c}\left(n_{c}+1\right)}}+\sqrt{\frac{n_{c}+n}{n n_{c}}}=\frac{n-1}{\sqrt{n(n+1)}}+\sqrt{\frac{2}{n}}
$$

which expresses the condition of equality between the two characteristic speeds in the bielliptic and Hohmann cases. $n_{c}^{*}$ is found to be the real root of eqn (78) other than $n_{c}=n$. Consequently, in the fixed-time case there exists a lower bound for the time parameter

$$
K_{1}^{*}=\frac{1}{2}\left\{\left(\frac{n_{c}^{*}+1}{n+1}\right)^{3 / 2}+\left(\frac{n_{c}^{*}+n}{n+1}\right)^{3 / 2}\right\} \quad 11.94<n \leqq 15.58,
$$

which is the transfer time along the bielliptic tangential trajectory, with $n_{c}=n_{c}^{*}$. From formula (79) we found that at the points:

$$
\begin{aligned}
& n=11.94: n_{c}^{*}=\infty, \quad K_{1}^{*}=\infty, \\
& n=15.58: n_{c}^{*}=15.58, \quad K_{1}^{*}=1.788 \text {. }
\end{aligned}
$$




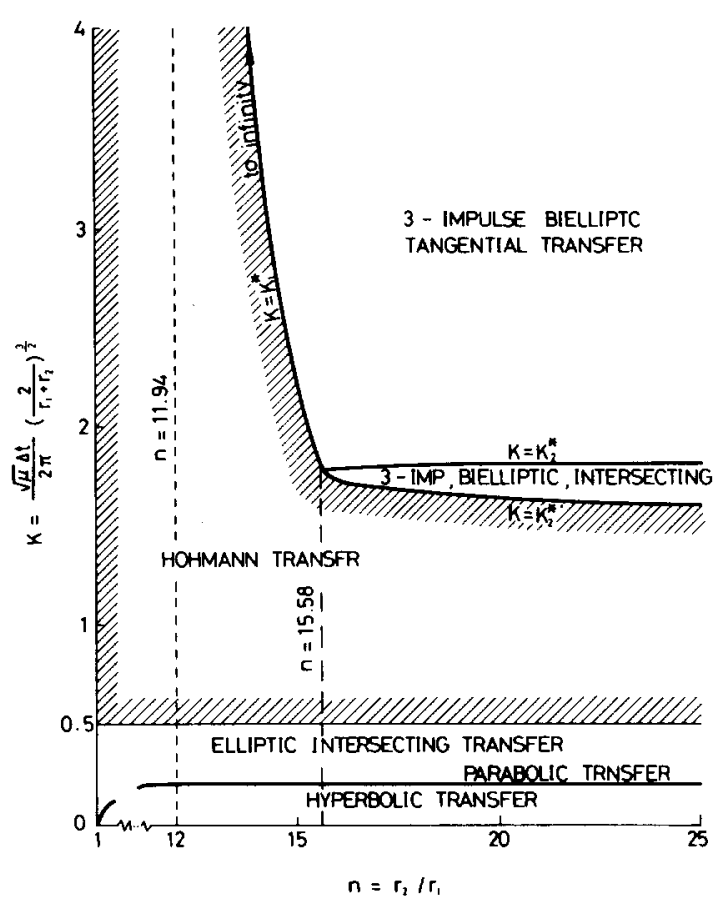

Fig. 9. Regions for optimal transfer.

When $n>15.58$, the higher the value of $n_{c}$, the better will be the fuel economy, hence on this range the minimum value of $n_{c}$ is $n$, and instead of eqn (79), we have the lower bound:

$$
K_{2}^{*}=\frac{1}{2}\left\{1+\left(\frac{2 n}{n+1}\right)^{3 / 2}\right\}
$$

The boundaries $K=K^{*}$ and $K=K_{2}^{*}$, as shown in Fig. 9, delimit the region in which Hoelker's bielliptic transfer satisfying the time constraint is better than the Hohmann transfer with delay.

It can be proved by perturbing the Hoelker's transfer that we can always find a three-impulse bielliptic intersecting transfer with the same time constraint while yielding a smaller characteristic speed. But the improvement is small and hence, when $K>K_{2}^{*}$ and $n>$ 15.58, Hoelker's bielliptic tangential transfer may be considered as the optimal. However, when $K<K_{2}^{*}$, Hoelker's transfer is no longer possible; it can be shown by the same analysis that there exists a new lower bound $K_{2}^{* \prime}$ such that, when $K_{2}^{* \prime}<K<K_{2}^{*}$, a three-impulse interesting bielliptic transfer satisfying the time constraint with smaller characteristic speed than the Hohmann transfer can be found. This new lower bound is given by:

$$
K_{2}^{* \prime}=\frac{1}{2}\left\{1+\left(\frac{2 n}{n+1}\right)^{3 / 2}\left[1-\frac{2}{\pi} \tan ^{-1}\left(\frac{(1-Z)(2-Z)}{2+Z}\right)^{1 / 2}\right]\right\}
$$

where

$$
Z=(3 n+1) \sqrt{\frac{2}{(n+1)^{3}}}
$$

Hence we may say that, for $n>15.58$ :

$K_{2}^{*^{\prime}} \leqq K<K_{2}^{*}$ : the optimal solution is intersecting bielliptic,

$0.5 \leqq K \leqq K_{2}^{* \prime}:$ the optimal solution is Hohmann.

The derivation of formulas (81), (82) is too long to be included here owing to the limited space.

To give a global view of the situation, the various possible optimal solutions in different regions in the $n$, $K$-plane is summarized in Table 2, and graphically

\begin{tabular}{|c|c|c|}
\hline $\begin{array}{l}\text { Distance Ratio } \\
n=r_{2} / r_{1}\end{array}$ & $\begin{array}{l}\text { Fixed Duration } \\
K=\Delta t \frac{\sqrt{\mu}}{2 \pi}\left(\frac{2}{r_{1}+r_{2}}\right)^{3 / 2}\end{array}$ & $\begin{array}{l}\text { Nature of the Optimal } \\
\text { Transfer Trajectory }\end{array}$ \\
\hline \multirow{3}{*}{$n \geq 1$} & $\mathrm{~K}<\mathrm{K}_{\mathrm{p}}^{*}$ & 2-impulse, hyperbolic \\
\hline & $K=K_{p}^{*}$ & 2-impulse, parabolic \\
\hline & $\mathrm{K}_{\mathrm{p}}^{*}<\mathrm{K}<0.5$ & $\begin{array}{l}\text { 2-impulse, elliptic, } \\
\text { nonHohmann }\end{array}$ \\
\hline $1 \leq n \leq 11.94$ & $K \geqq 0.5$ & 2-impulse, elliptic, Hohmann \\
\hline \multirow{2}{*}{$11.94<\mathrm{n}<15.58$} & $0.5<K<K_{1}^{*}$ & 2-impulse, elliptic, Hohmann \\
\hline & $\mathrm{K}>\mathrm{K}_{1}^{*}$ & $\begin{array}{l}\text { 3-impulse, bielliptic, } \\
\text { tangential }\end{array}$ \\
\hline \multirow{3}{*}{$\mathrm{n} \geqq 15.58$} & $0.5 \leqq K<K_{2}^{*}$ & 2-impulse, elliptic, Hohmann \\
\hline & $K_{2}^{* \prime} \leqq K<K_{2}^{*}$ & $\begin{array}{l}3 \text {-impulse, bielliptic, } \\
\text { intersecting }\end{array}$ \\
\hline & $\mathrm{K} \geqq \mathrm{K}_{2}^{*}$ & $\begin{array}{l}\text { 3-impulse, bielliptic, } \\
\text { tangential }\end{array}$ \\
\hline
\end{tabular}
depicted in Fig. 9.

Table 2. Nature of optimal transfer in $n, K$-regions 


\section{REFERENCES}

1. J. P. Marec, Optimal Space Trajectories, pp. 275-305. Elsevier, Amsterdam (1979).

2. R. H. Battin, A new transformation invariant in the orbital boundary value problem. J. Guidance and Control, 1, 50-55 (1978).

3. Th. Godal, Method for determining the initial velocity vector corresponding to a given time of free flight transfer between given points in a simple gravitational field. Astronautik 2 , $183-186(1 \% 1)$.

4. F. T. Sun, On the orbital isochronism. Celestial Mech. 3, 272-288 (1971).

5. F. T. Sun, On the minimum time trajectory and multiple solutions of Lambert's problem. AAS/AIAA Astrodynamics Conf., Provincetown, Mass. (1979).

6. F. T. Sun, A new treatment of Lambertian mechanics. Acta Astronautica 8, 105-122 (1981).

7. D. J. Jezewski, A method for determining optimal fixed-time, $\mathrm{N}$-impulse trajectories between arbitrary inclined orbits. 19th IAF Cong., New York, 1968.
8. P. M. Lion, Primer vector on fixed-time impulsive trajectories. AIAA J. 6, 127-132 (1968).

9. J. P. Peltier, Some impulsive rendezvous trajectories and their possible optimality. AIAA J. 10, 440-446 (1972).

10. G. M. Levine, Application of midcourse guidance technique to orbit determination. AIAA J. 3, 137-139 (1965).

11. N. X. Vinh, Invariance in the Lambert's problems. Lecture Notes, The University of Michigan (1977).

12. F. T. Sun, A global analysis of two-terminal trajectories. Acta Astronautica 4, 469-493 (1977).

13. R. F. Hoelker and R. Silber, The bielliptical transfer between coplanar circular orbits. Ballistic Missiles and Space Technology III, 164-175 (1961).

14. R. H. Battin, Lambert problem revisited. AIAA J. 15, 707713 (1977).

15. W. R. Hamilton, Hodographic and anthodographic isochronism. In Mathematical Papers (Edited by Sir William R. Hamilton), Vol II, Note 8, p. 630. Royal Irish Academy (1940). 